\title{
The clinical significance of Notch 1 immunoexpression in Caucasian patients with colorectal adenocarcinoma
}

\author{
Adam Piecuch ${ }^{1}$, Marek Kucharzewski², Józef Kurek ${ }^{3}$, Marek Michalski ${ }^{1}$, Dawid Jasiński ${ }^{1}$, \\ Marlena Brzozowa-Zasada ${ }^{1}$ \\ ${ }^{1}$ Department of Histology and Cell Pathology, School of Medicine with the Division of Dentistry in Zabrze, Medical University \\ of Silesia in Katowice, Poland \\ 2Department of Descriptive and Topographical Anatomy, School of Medicine with the Division of Dentistry in Zabrze, \\ Medical University of Silesia in Katowice, Poland \\ ${ }^{3}$ Department of General, Endocrinological and Oncological Surgery, Municipal Hospital, Jaworzno, Poland
}

Gastroenterology Rev 2020; 15 (4): 314-322

DOI: https://doi.org/10.5114/pg.2020.101560

Key words: colorectal adenocarcinoma, Notch1, five-year overall survival.

Address for correspondence: Dr. Marlena Brzozowa-Zasada, Department of Histology and Cell Pathology, School of Medicine with the Division of Dentistry, Medical University of Silesia, 19 Jordana St, 41-808 Zabrze, Poland, e-mail: marlena.brzozowa@op.pl

\begin{abstract}
Introduction: Colorectal cancer (CRC) is traditionally regarded as the most commonly diagnosed gastrointestinal malignant disease. Nevertheless, despite advances in diagnosis and novel therapeutic options, the clinical outcomes of patients are still not satisfactory.

Aim: To investigate the clinicopathological and prognostic roles of Notch1 expression, the immunohistochemical investigation was performed in samples of CRC tumour tissues, adjacent non-pathological mucosa, and metastatic foci in regional lymph nodes in Caucasian patients.

Material and methods: Paraffin-embedded adenocarcinoma samples were assessed immunohistochemically for Notch1 protein and scored according to the percentage of cells with a positive reaction combined with staining intensity. Connections between Notch1 immunoexpression and clinicopathological factors including the 5-year overall survival (OS) were evaluated.

Results: The level of the Notch1 immunohistochemical reactivity was correlated with the grade of the histological differentiation, size of the primary tumour, regional lymph node involvement, and perineural invasion (all $p<0.001$ ). Kaplan-Meier survival analysis showed that the survival time for patients with a low expression of Notch1 was significantly longer than that for patients with moderate or strong level of Notch1 immunoreactivity $(p<0.001)$.

Conclusions: The enhanced level of Notch1 immunoexpression was significantly associated with malignancy-related clinicopathological factors and reduced the 5 -year overall survival in CRC patients.
\end{abstract}

\section{Introduction}

Colorectal cancer (CRC) is traditionally regarded as the most commonly diagnosed gastrointestinal malignant disease. Nevertheless, despite advances in diagnosis and novel therapeutic options, the clinical outcomes of CRC patients with locally advanced or metastatic disease are still not satisfactory. The patients' overall survival (OS) is largely dependent on the disease stage at the time of diagnosis and/or surgical resection $[1,2]$. Importantly, approximately $20 \%$ of the diagnoses are made in the metastatic stage of the disease. In this case the 5 -year overall survival is less than $15 \%$. Importantly, the prognoses are not satisfactory even in the case of patients with primary tumours $[3,4]$. Therefore, the identification of novel approaches for describing patients from the high-risk group, particularly in the context of clinical outcomes, has become a major challenge $[5,6]$.

The Notch signalling pathway is an evolutionarily conserved cell signalling mechanism involved in a wide array of cellular processes. Among them should be 
mentioned proliferation, differentiation, apoptosis, and stem cell maintenance [7-9]. It is generally accepted that there are four Notch receptors: Notch1, 2, 3, and 4, which are synthesised as precursors composed of extracellular, transmembrane, and intracellular domains. Within the cisterns of Golgi complex, the precursors of Notch receptors are cleaved by furin-like convertases to generate two subunits. One subunit contains most of the extracellular domain, and the second subunit consists of the rest of the extracellular and transmembrane domains. The Notch ligands comprise five members: Jagged1/2 and Delta-like 1/3/4 (DLL1/3/4). All these ligands are single-pass type I transmembrane proteins. The extracellular domain of the Notch receptor has been shown to contain 36 EGF-like repeats [10, 11]. Ligand binding to EGF-like repeats unfolds the negative regulatory region (NRR), permitting the next cleavage by metalloproteases of the ADAM family [11]. During the next step, $\gamma$-secretase complex executes an intramembrane cleavage releasing the Notch intracellular domain (NotchIC or NICD), which translocates to the nucleus [10]. It has been demonstrated that for activation of The Notch signalling the Mastermind-like family of proteins (MAML1/2/3) are needed. MAML forms a ternary complex with CBF1 (Centromere-binding protein 1) NotchIC by direct interaction with NotchIC. Then, the ternary complex composed of CBF1-NotchIC-MAML acts as a transcriptional activator, leading to Notch target gene transcription. Among the primary targets there are several genes belonging to the basic helix-loop-helix (bHLH) family. Following Notch activation, at least two families of bHLH proteins are induced: the Hairy/ Enhancer-of-Split (HES) family and the Hairy-Related Transcription factor (HRT) family, which are known to be transcriptional repressors [12].

Notch signalling has been revealed to be involved in the pathogenesis of gastrointestinal tumours such as gastric and colon cancer. Similarly as in the differentiation process, its effect during carcinogenesis is probably cell type specific and circumstance dependent. Notch proteins suppress malignant transformation in some tissues and promote metastatic events in others. An improved understanding of the Notch-mediated signalling pathway in tissues under physiological conditions as well as in those undergoing malignant transformation is significant from the clinical point of view because it may lead to the development of promising cancer therapeutics [7-9, 13]. Expression of Notch1 protein has been revealed to be upregulated in CRC patients. However, it should be noted that studies by Zhang et al. and Chu et al. have been conducted on Chinese patients (Asians) with CRC $[14,15]$. Several reports indicate that there are racial disparities in CRC biology and behaviour
[4-6]. Thus, it remains to be determined whether those findings are applicable to the general population with CRC. The current study investigated the expression of Notch1 protein in colorectal adenocarcinoma samples to assess its prognostic significance by correlating its expression with the clinicopathological variables and survival of Caucasian patients.

\section{Material and methods}

\section{Tissue samples}

The study was conducted on formalin-fixed and paraffin-embedded colorectal adenocarcinoma samples archived in the files of the Department of Pathomorphology in Zabrze (Poland). Tissue specimens were received from 92 colorectal adenocarcinoma Polish (Caucasian) patients who underwent surgical resection on the Surgical Clinics in 2011. The exclusion criteria were as follows: (1) history of previous malignant disease, (2) familial adenomatous polyposis, (3) inflammatory bowel disease, (4) preoperative anti-cancer treatment, and (5) evidence of distant metastasis. The clinicopathological characteristics obtained from the medical records were as follows: age, gender, location of tumour, grade of tumour differentiation, depth of invasion, tumour size, presence or absence of angio- and perineural invasion, lymph node metastasis, operation record, treatment record, reoccurrence, and vital status at the last follow-up date.

The specimens belonged to 46 men and 46 women (mean age: 62.5 ; range $48.5-71.5$ years). Tumours were located in the proximal part of the colon 30 (32.60\%) cases, in the distal part of the colon 31 (33.70\%), and in the rectum 31 (33.70\%). Three levels of differentiation were used to classify the grading as follows: well differentiated (G1), 35 (38.04\%) cases; moderately differentiated (G2), 34 (36.96\%) cases, and poorly differentiated (G3), 23 (25\%) cases (Table I).

\section{Immunohistochemical staining}

For the immunohistochemical studies the paraffin-embedded specimens were cut into 4- $\mu$ m-thick sections, fixed on Polysine slides and deparaffinised in xylene and rehydrated through a graded series of alcohol. To retrieve the antigenicity, the tissue sections were treated twice with microwaves in a $10 \mathrm{mM}$ citrate buffer ( $\mathrm{pH}$ 6.0) for 8 min each. Subsequently, sections were incubated with rabbit polyclonal antibody to Notch1 (final dilution 1 : 400) (Abcam cat. number ab27526). For visualisation of protein expression the sections were treated with the BrightVision detected system and Permanent AP Red Kit (Zytomed). Mayer's haematoxylin was used to counterstain the nuclei. 
Table I. Demographic, clinical, and tumour-related characteristics of patients included in the study $(n=92)$

\begin{tabular}{|c|c|c|}
\hline Parameter & $N$ & $\%$ \\
\hline \multicolumn{3}{|l|}{ Age [years]: } \\
\hline$\leq 62$ & 46 & 50.00 \\
\hline$\geq 63$ & 46 & 50.00 \\
\hline$M \pm S D$ & \multicolumn{2}{|c|}{$60.53 \pm 13.79$} \\
\hline Me (Q1-Q3) & \multicolumn{2}{|c|}{$62.5(48.5-71.5)$} \\
\hline Min.-max. & \multicolumn{2}{|c|}{$34-87$} \\
\hline \multicolumn{3}{|l|}{ Gender: } \\
\hline Females & 46 & 50.00 \\
\hline Males & 46 & 50.00 \\
\hline \multicolumn{3}{|c|}{ Location of tumour: } \\
\hline Proximal colon & 30 & 32.60 \\
\hline Distal colon & 31 & 33.70 \\
\hline Rectum & 31 & 33.70 \\
\hline \multicolumn{3}{|c|}{ Grade of histological differentiation: } \\
\hline G1 & 35 & 38.04 \\
\hline $\mathrm{G} 2$ & 34 & 36.96 \\
\hline G3 & 23 & 25.00 \\
\hline \multicolumn{3}{|l|}{ Depth of invasion: } \\
\hline $\mathrm{T} 1 / \mathrm{T} 2$ & 49 & 53.26 \\
\hline $\mathrm{T} 3 / \mathrm{T} 4$ & 43 & 46.74 \\
\hline \multicolumn{3}{|c|}{ Size of primary tumour [cm]: } \\
\hline$\leq 9$ & 46 & 50.00 \\
\hline$\geq 10$ & 46 & 50.00 \\
\hline$M \pm S D$ & \multicolumn{2}{|c|}{$9.48 \pm 3.64$} \\
\hline Me (Q1-Q3) & \multicolumn{2}{|c|}{$9.5(6-12)$} \\
\hline Min.-max. & \multicolumn{2}{|c|}{$3-17$} \\
\hline \multicolumn{3}{|l|}{ Angioinvasion: } \\
\hline No & 43 & 46.74 \\
\hline Yes & 49 & 53.26 \\
\hline \multicolumn{3}{|c|}{ Regional LN involvement: } \\
\hline No & 43 & 46.74 \\
\hline Yes & 49 & 53.26 \\
\hline \multicolumn{3}{|l|}{ Perineural invasion: } \\
\hline No & 49 & 53.26 \\
\hline Yes & 43 & 46.74 \\
\hline
\end{tabular}

\section{Semi-quantitative analysis of Notch1 expression in colorectal adenocarcinoma samples}

The scores were assigned separately for the stained area and for the intensity of the immunohistochemical reaction. Quantification connected to the stained area of the tissue section was performed as follows: (1) $<33 \%$ of cells showed immunoreaction, (2) 33$66 \%$ of the cells had positive reaction to Notch1, and (3) $>66 \%$ of the cells were positive. The intensity of the immunohistochemical reaction was quantified as follows: (1) absent or weak, (2) moderate, and (3) strong. Each tissue section was characterised by a final grade derived from the multiplication of the stained area and the intensity of the staining. The Notch1 expression was considered to be absent/low for grade 1 ; moderate for grades 2, 3, and 4, and strong for grades 6 and 9 .

\section{Survival analysis}

A survival analysis was conducted in 92 patients. The survival curves were generated using the Kaplan-Meier method. The overall survival (OS) was defined as the length of time between surgery and death. The follow-up period was 60 months. Patients alive were censored at 5 years.

\section{Statistical analysis}

Statistical analyses were conducted using Statistica 9.1 (StatSoft, Poland). The clinical characteristics of the patients in relation to Notch1 immunoreactivity were assessed by performing the Kruskal-Wallis test and U Mann-Whitney test. Additionally using Spearman's rank correlation coefficient, the relationship between the immunoexpression of Notch1 and age, grade of tumour differentiation, and size of primary tumour were assessed. The Kaplan- Meier Method was used to study survival curves and the long-rank test to compute differences between the curves.

\section{Results}

To investigate the clinicopathological and prognostic roles of Notch1 expression, the immunohistochemical analysis was performed in CRC tumour tissues, adjacent non- pathological mucosa, and metastatic foci in lymph nodes (Figure 1). It should be noted that only trace expression of this protein was revealed in adjacent non-tumour colorectal mucosa, whereas the high expression was demonstrated in well, moderately, and poorly differentiated tumours (Figures 1 and 2). Among the 92 samples, 33 showed a strong immunohistochemical reaction, 21 demonstrated moderate immunoreactivity, 29 (31.52\%) revealed weak expression, and nine were characterised as negative in the context of Notch1 immunoexpression. Moreover, moderate and strong expression was demonstrated in metastatic foci in lymph nodes.

The relationships between the Notch1 levels and each clinicopathological parameter are summarised in Table II. As demonstrated, the level of the Notch1 

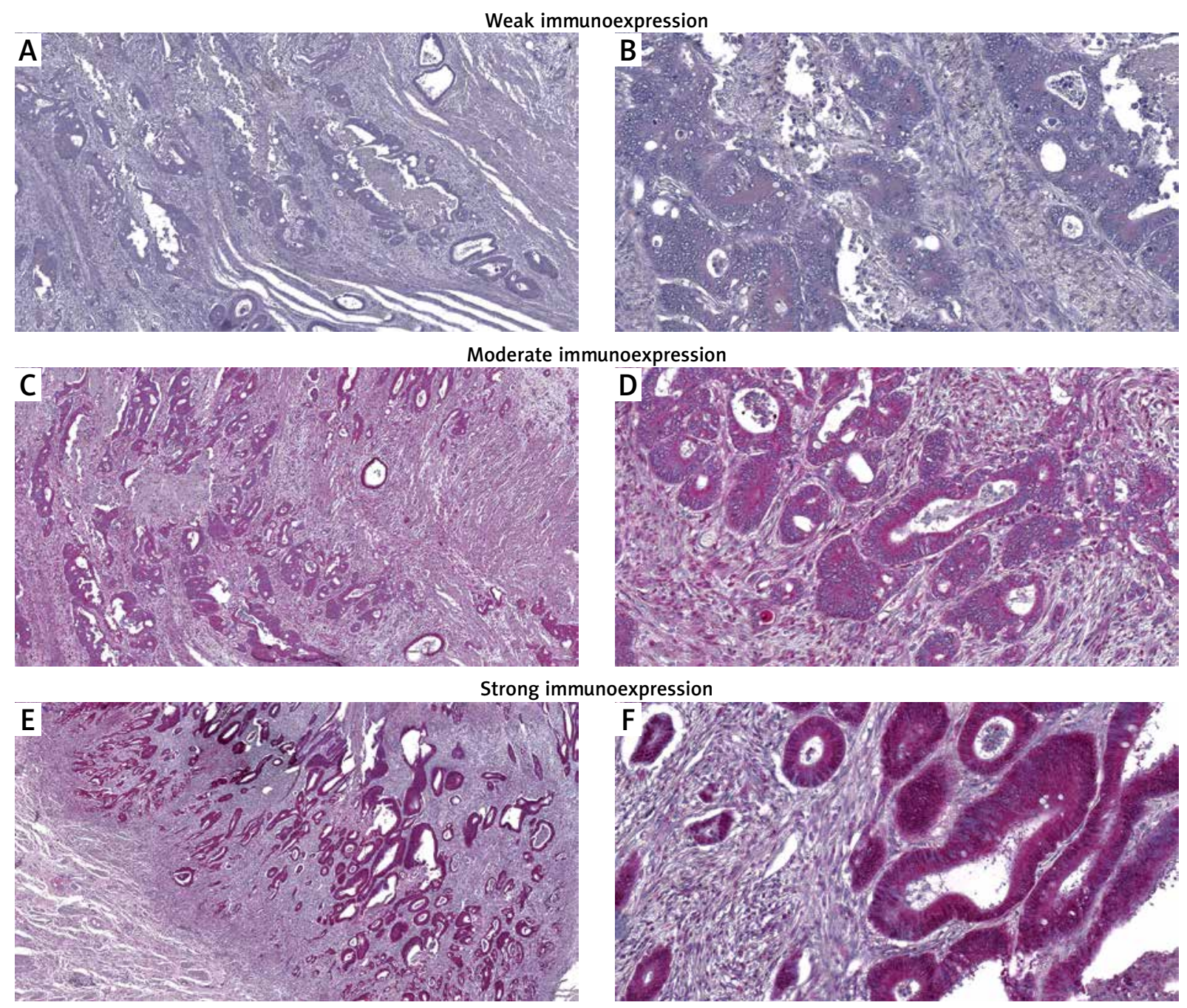

Figure 1. Immunohistochemical expression of Notch1 protein in tumour tissue: A, B - weak expression of Notch1 protein in colon adenocarcinoma samples, C, D - moderate expression of Notch1, E, F - strong expression of Notch1 (A, C, E-25x; B, D, F-200x)

immunohistochemical reactivity was correlated with the grade of the histological differentiation $(p<0.001)$. A strong expression of the Notch 1 protein was detected more frequently in patients with G3 tumours $(p<0.001)$. A significant correlation was also observed between the strong immunoreactivity of the Notch 1 protein and larger tumour size $(p<0.001)$. The expression of this protein increased with an increase in the tumour size $(p<0.001)$. The other clinicopathological variables associated with the immunoexpression of Notch1 included regional lymph node metastasis and perineural invasion. The patients with regional lymph node involvement were characterised by strong Notch1 immunoreactivity $(p<0.001)$. In samples of patients with perineural invasion, the expression of the Notch1 protein was also revealed to be strong ( $p<0.001$; Table II). The statistical evaluation of Notch1 immunoex-

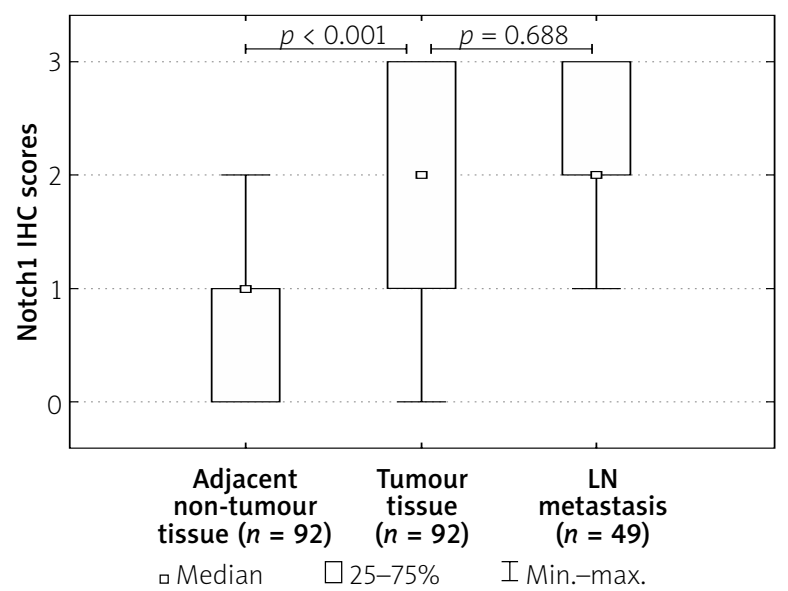

Figure 2. Immunoexpression of Notch1 in colorectal cancer tumour tissues, adjacent non-tumour tissues, and metastatic foci in lymph nodes 
Table II. Correlations between Notch1 immunoexpression and clinicopathological characteristics in colorectal cancer patients

\begin{tabular}{|c|c|c|c|c|c|}
\hline \multirow[t]{2}{*}{ Parameter } & & \multicolumn{3}{|c|}{ The immunoexpression level of Notch1 } & \multirow[t]{2}{*}{ Statistical analysis } \\
\hline & & Low & Moderate & Strong & \\
\hline \multirow[t]{4}{*}{ Age [years] } & \multirow[t]{2}{*}{$\leq 62$} & 21 & 8 & 17 & \multirow{4}{*}{$\begin{array}{c}Z=-0,373 ; \mathrm{NS} \\
R=0.044\end{array}$} \\
\hline & & $45.65 \%$ & $17.39 \%$ & $36.96 \%$ & \\
\hline & \multirow[t]{2}{*}{$>63$} & 17 & 13 & 16 & \\
\hline & & $36.96 \%$ & $28.26 \%$ & $34.78 \%$ & \\
\hline \multirow[t]{4}{*}{ Gender } & \multirow[t]{2}{*}{ Female } & 17 & 12 & 17 & \multirow[t]{4}{*}{$Z=0.905 ; \mathrm{NS}$} \\
\hline & & $36.96 \%$ & $26.09 \%$ & $36.96 \%$ & \\
\hline & \multirow[t]{2}{*}{ Male } & 21 & 9 & 16 & \\
\hline & & $45.65 \%$ & $19.57 \%$ & $34.78 \%$ & \\
\hline \multirow[t]{6}{*}{ Location of tumour } & \multirow[t]{2}{*}{ Proximal colon } & 12 & 7 & 11 & \multirow{6}{*}{$\begin{array}{c}H(2.92)=0.269 \\
\text { NS }\end{array}$} \\
\hline & & $40.00 \%$ & $23.33 \%$ & $36.67 \%$ & \\
\hline & \multirow[t]{2}{*}{ Distal colon } & 12 & 10 & 9 & \\
\hline & & $38.71 \%$ & $32.26 \%$ & $29.03 \%$ & \\
\hline & \multirow[t]{2}{*}{ Rectum } & 14 & 4 & 13 & \\
\hline & & $45.16 \%$ & $12.90 \%$ & $41.94 \%$ & \\
\hline \multirow{6}{*}{$\begin{array}{l}\text { Grade of histological } \\
\text { differentiation }\end{array}$} & \multirow[t]{2}{*}{ G1 } & 24 & 6 & 5 & \multirow{6}{*}{$\begin{array}{c}H(2.92)=25.906 \\
p<0.001 \\
R=0.521\end{array}$} \\
\hline & & $68.57 \%$ & $17.14 \%$ & $14.29 \%$ & \\
\hline & \multirow[t]{2}{*}{ G2 } & 14 & 8 & 12 & \\
\hline & & $41.18 \%$ & $23.53 \%$ & $35.29 \%$ & \\
\hline & \multirow[t]{2}{*}{ G3 } & 0 & 7 & 16 & \\
\hline & & $0.00 \%$ & $30.43 \%$ & $69.57 \%$ & \\
\hline \multirow[t]{4}{*}{ Depth of invasion } & \multirow[t]{2}{*}{$\mathrm{T} 1 / \mathrm{T} 2$} & 23 & 11 & 15 & \multirow[t]{4}{*}{$Z=-1.477 ; \mathrm{NS}$} \\
\hline & & $46.94 \%$ & $22.45 \%$ & $30.61 \%$ & \\
\hline & \multirow[t]{2}{*}{$\mathrm{T} 3 / \mathrm{T} 4$} & 15 & 10 & 18 & \\
\hline & & $34.88 \%$ & $23.26 \%$ & $41.86 \%$ & \\
\hline Size of primary & $\leq 9$ & 30 & 8 & 8 & $Z=-4.010 ; p<0.001$ \\
\hline & & $65.22 \%$ & $17.39 \%$ & $17.39 \%$ & 0.415 \\
\hline & $>10$ & 8 & 13 & 25 & \\
\hline & & $17.39 \%$ & $28.26 \%$ & $54.35 \%$ & \\
\hline Angioinvasion & No & 20 & 11 & 12 & $Z=-1.337 ;$ NS \\
\hline & & $46.51 \%$ & $25.58 \%$ & $27.91 \%$ & \\
\hline & Yes & 18 & 10 & 21 & \\
\hline & & $36.73 \%$ & $20.41 \%$ & $42.86 \%$ & \\
\hline Regional LN & No & 32 & 10 & 1 & $Z=-6.547 ; p<0.001$ \\
\hline Involvement & & $74.42 \%$ & $23.26 \%$ & $2.33 \%$ & \\
\hline & Yes & 6 & 11 & 32 & \\
\hline & & $12.24 \%$ & $22.45 \%$ & $65.31 \%$ & \\
\hline Perineural invasion & No & 34 & 12 & 3 & $Z=-6.235 ; p<0.001$ \\
\hline & & $69.39 \%$ & $24.49 \%$ & $6.12 \%$ & \\
\hline & Yes & 4 & 9 & 30 & \\
\hline & & $9.30 \%$ & $20.93 \%$ & $69.77 \%$ & \\
\hline
\end{tabular}

$H$ - Kruskal- Wallis test, Z - U Mann-Whitney test, $R$-Spearman's rank correlation coefficient. 
pression according to age, sex, tumour location, depth of invasion, and angioinvasion revealed no significant difference among these variables (all $p>0.05$; Table II).

The Kaplan-Meier survival analysis showed that the overall survival rate in the group of patients with low expression level of Notch1 was significantly longer than that for patients with moderate or strong level of Notch1 immunoreactivity (Figure 3). The 5-year overall survival for patients with a low, moderate, or strong level of Notch1 immunoexpression was $68.4 \%, 14.3 \%$, and $12.1 \%$, respectively. The low-Notch 1 patients had an average survival time of 56.5 months ( $95 \%$ Cl: $54.401-$ 58.547), whereas the moderate-Notch1 expression and strong-Notch1 expression groups had an average survival time of 31.4 (95\% Cl: 23.12-39.82) and 22.4 months (95\% Cl: 16.86-27.98), respectively. The average survival time for all of the patients was 38.5 months $(95 \% \mathrm{Cl}$ : 34.28-42.82; Table III).

In the univariate analysis the age, grade of tumour differentiation, depth of tumour invasion, size of primary tumour, angioinvasion, regional lymph node involvement, perineural invasion, and Notch1 immunoexpression were found to be significantly associated with reduced 5-year survival time. Nevertheless, multivariate analysis demonstrated that only age $(p=0.030)$, grade of tumour differentiation $(p<0.001)$, angioinvasion $(p=0.002)$, and regional lymph node involvement $(p=0.049)$ were independent risk factors for worse survival (Table IV).

\section{Discussion}

As revealed by studies, Notch receptors are known to be highly oncogenic in the case of CRC. Zhang et al. demonstrated that overexpression of Notch1 was clearly associated with proliferation, colony formation, cell cycling, and tumosphere formation of colon cancer cells under in vitro conditions. Furthermore, Notch 1 was also responsible for the development and increased growth of implanted colon cancers in vivo [14]. Expression of Notch1 has been positively associated with depth of in-

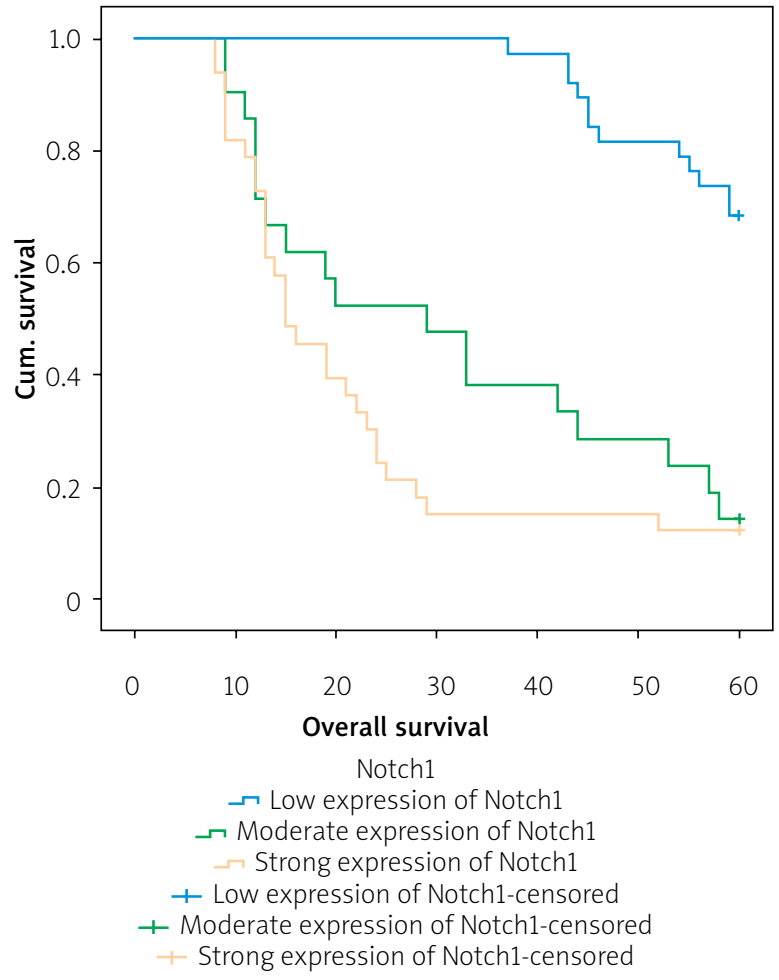

Figure 3. Kaplan-Meier survival curves of colorectal cancer patients with different expression of Notch1 protein; follow-up period $=60$ months

vasion, lymph node metastases, and tumour-node-metastasis (TNM) stage [15]. It is probably connected with the role of Notch1 in EMT (Epithelial- Mesenchymal Transition) induction [9]. Fender et al. demonstrated that retroviral transduction of constitutively active ICN1 (intracellular domain of Notch1) into colon cancer cell line HCT-116 was associated with enhanced level of EMT and increased expression of such proteins as CD44, Slug, and Smad-3 [16]. It should be also mentioned that Notch may crosstalk with other signalling

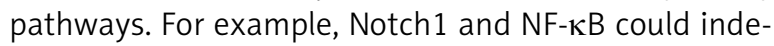
pendently contribute to tumour progression, but their

Table III. Means and medians for survival time of colorectal cancer patients according to Notch1 immunoexpression

\begin{tabular}{|c|c|c|c|c|c|c|c|c|}
\hline \multirow[t]{3}{*}{ Notch1 } & \multicolumn{4}{|c|}{ Mean } & \multicolumn{4}{|c|}{ Median } \\
\hline & \multirow[t]{2}{*}{ Estimate } & \multirow[t]{2}{*}{ Std. error } & \multicolumn{2}{|c|}{$95 \%$ confidence interval } & \multirow[t]{2}{*}{ Estimate } & \multirow[t]{2}{*}{ Std. error } & \multicolumn{2}{|c|}{$95 \%$ confidence interval } \\
\hline & & & Lower bound & Upper bound & & & Lower bound & Upper bound \\
\hline Grade $0-1$ & 56.474 & 1.058 & 54.401 & 58.547 & . & . & . & \\
\hline Grade 2 & 31.476 & 4.261 & 23.125 & 39.827 & 29.000 & 8.010 & 13.300 & 44.700 \\
\hline Grade 3 & 22.424 & 2.835 & 16.868 & 27.981 & 15.000 & 2.392 & 10.311 & 19.689 \\
\hline Overall & 38.554 & 2.180 & 34.282 & 42.827 & 44.000 & 9.110 & 26.145 & 61.855 \\
\hline
\end{tabular}


Table IV. Univariate and multivariate analyses of various prognostic parameters in colorectal cancer patients using Cox regression analyses

\begin{tabular}{|c|c|c|c|c|}
\hline \multirow[t]{2}{*}{ Prognostic parameter } & Univariate analysis & \multicolumn{3}{|c|}{ Multivariate analysis } \\
\hline & $P$-value & HR & $95 \% \mathrm{Cl}$ & $P$-value \\
\hline Age (> 62 vs. $\leq 62)$ & 0.035 & 1.875 & $1.063-3.307$ & 0.030 \\
\hline Gender (male vs. female) & NS & - & - & - \\
\hline Grade & $<0.001$ & 17.133 & $6.673-43.990$ & $<0.001$ \\
\hline Depth of invasion & 0.020 & 0.793 & $0.403-1.560$ & NS \\
\hline Size of primary tumour (> 9 vs. $\leq 9$ ) & $<0.001$ & 1.967 & $0.847-4.568$ & NS \\
\hline Angioinvasion (yes vs. no) & 0.003 & 0.208 & $0.077-0.559$ & 0.002 \\
\hline Regional LN involvement (yes vs. no) & $<0.001$ & 2.745 & $1.006-7.491$ & 0.049 \\
\hline Perineural invasion (yes vs. no) & $<0.001$ & 0.982 & $0.428-2.250$ & NS \\
\hline Notch1 immunoexpression & $<0.001$ & 1.681 & $0.941-3.002$ & NS \\
\hline
\end{tabular}

interaction is thought to be a determinant that would affect the clinical outcome of the disease and therapeutic intervention of CRC patients [17]. Kim et al. found that Notch1 was able to suppress the expression of Wnt target genes even when $\beta$-catenin destruction by the adenomatous polyposis coli (APC) complex had been disrupted. Induction of Notch1 transformed high-grade adenoma into low-grade adenoma in an APC ${ }^{\text {Min }}$ mouse model of colon cancer and suppressed the expression of Wnt target genes [18]. Microarray analysis revealed a negative correlation between the Notch1 target gene, Notch-regulated ankyrin repeat protein 1 (NRARP1), and Wnt target genes [18].

In the current study a high expression of Notch1 protein was observed in primary colorectal adenocarcinoma foci and metastatic foci in LNs (lymph nodes). Moreover, the high level of Notch1 immunoexpression in tumour tissues was demonstrated to be strongly correlated with malignancy-related clinicopathological factors and 5-year OS of Caucasian patients. It should be pointed out that the high expression of Notch1 was significantly associated with advanced histological tumour grade. The Kruskal-Wallis test showed a statistical difference between the patients with G1 tumours and those with G2 tumours, and between the patients with G1 tumours and those with G3 tumours. In both cases, patients with $\mathrm{G} 1$ tumours were characterised by a low level of Notch1 expression $(R=0.521 ; p<0.001)$. The $U$ Mann-Whitney test and Spearman's rank correlation coefficient demonstrated that strong Notch1 expression was related to increased tumour size and the presence of perineural invasion, suggesting that this protein might participate in malignant formation and tumour progression [9]. It should be mentioned that similar results have been obtained by Zhang et al. and Chu et al. in Chinese patients with CRC $[14,15]$. The high expression of Notch1 in metastatic foci in lymph nodes may indicate that colorectal cancer cells with such expression are characterised by enhanced capacity for metastasis formation. It should also be mentioned that enhanced level of Notch1 immunoexpression was significantly associated with worse overall survival in CRC patients. Nevertheless, a multivariate analysis demonstrated that Notch1 was not revealed as an independent risk factor for CRC prognosis.

In gastric cancer, the Notch1 expression appeared in tissues with intestinal metaplasia and well-differentiated intestinal type of gastric cancer. Notch1 may play a crucial role in both promoting metaplastic transition of gastric epithelial cells and in maintaining constant proliferation of intestinalised epithelial cells $[19,20]$. The activated form of the Notch 1 receptor has promoted colony-forming ability and xenografted tumour growth of human stomach adenocarcinoma SC-M1 cells $[21,22]$. Activation of the Notch1 signalling pathway could promote progression of the disease at least in part by cyclooxyhenase-2 (COX-2) [23, 24]. Interestingly, the colony forming, migration, and invasive abilities enhanced through N1IC were suppressed in SC-M1 cells after treatment with the COX-2 inhibitor NS-398 or COX-2 knockdown. These processes were restored by prostaglandin PGE2 or exogenous cox-2 [24].

Notch 1 expression is also connected with breast cancer development. Rizzo et al. reported that $80 \%$ of epithelial hyperplasias of usual type (HUTs), $67 \%$ of ductal carcinomas in situ (DCISs), 89\% of invasive ductal carcinomas (IDCs), and $57 \%$ of invasive lobular carci- 
nomas (ILCS) revealed high expression of Notch1, while expression in the non-pathological samples was characterised as low or negative [25]. The high level of Notch1 was also revealed in ovarian cancer cell lines OVCAR3, SKOV3, and CaOV3. siRNA downregulation of NICD1 in those cells resulted in inhibition of cell proliferation. Notch 1 also seems to be a very interesting factor in the context of chemoresistance [26-29]. Dysregulation of miRNA altered a network of functional targets and signalling pathways resulting in acquired chemoresistance in human cancers. Liu et al. demonstrated that increased expression of miR-199b-5p sensitised ovarian cancer cells to cisplatin-induced cytotoxicity. Conversely, re-expression of miR-199b-5p and siRNA-mediated JAG1 knockdown or treatment with GSI attenuated enhanced cisplatin-mediated cell cytotoxicity. It seems, therefore, that the epigenetic silencing of miR-199b-5p was significantly associated with acquired chemoresistance in ovarian cancer cells through the activation of JAG1-Notch1 [30]. Zhou et al. provided evidence that downregulation of miR-449a could enhance ovarian cancer cell proliferation and induce cisplatin resistance. Importantly, inhibition of Notch 1 signalling by overexpression of miR-449a could sensitise chemoresistant ovarian cancer cells to cisplatin-induced cytotoxicity. A high level of Notch1 has also been clearly correlated with poor clinical prognosis in patients with lung adenocarcinoma [31]. Induction of Notch signalling through Notch 1 upregulation has been characterised in $30 \%$ of primary human NSCLC [32]. Allen et al. revealed that in the mouse alveolar epithelium, acute activation of NICD1 resulted in a wave proliferation and the formation of extensive hyperplasia, but the bulk of the alveolar hyperplasia was cleared from the lung periphery [33]. In the study by Yuan et al. a combined analysis of 19 eligible clinical studies demonstrated a predictive value for Notch 1 and Notch3 expression in NSCLC patients. The results of the meta-analysis suggested that Notch 1 is characterised as highly expressed in lung cancer compared with non-pathological tissue and is associated with lymph node metastasis and TNM stage. This may suggest that overexpression of Notch1 might have a prognostic value for overall survival [34].

\section{Conclusions}

The results of our study revealed a correlation between strong Notch1 immunoexpression and advanced histological tumour grade. Moreover, the high level of Notch1 immunoexpression was correlated with increased tumour size, the presence of perineural invasion, and reduced 5-year OS. A multivariate analysis revealed that the independent risk factors for CRC prognosis were age, grade of tumour differentiation, angioinvasion, and regional lymph node involvement. Interestingly, Notch1 was not revealed as an independent risk factor for Caucasian patients with CRC.

\section{Acknowledgments}

This study was supported by grant KNW-2-B30/ D/6/N and KNW-1-004/K/8/O of the Medical University of Silesia.

\section{Conflict of interest}

The authors declare no conflict of interest.

\section{References}

1. Muller MF, Ibrahim AE, Arends MJ. Molecular pathological classification of colorectal cancer. Virchovs Arch 2016; 469: 125-34.

2. Ahmad S, Johnson K, Ahmed O, Iqbal N. Advances in the management of colorectal cancer: from biology to treatment. Int J Colorectal Dis 2014; 29: 1031-42.

3. Mody K, Bekaii-Saab T. Clinical Trials and progress in metastatic colon cancer. Surg Oncol Clin N Am 2018; 27: 349-65.

4. Alwers E, Jia M, Kloor M, et al. Associations between molecular classifications of colorectal cancer and patient survival: a systematic review. Clin Gastroenterol Hepatol 2019; 17: 402-10.

5. Grizzle WE, Manne U, Weiss HL, et al. Molecular staging of colorectal cancer in African-American and Caucasian patients using phenotypic expression of p53, Bcl-2, MUC-1 and p27(kip1). Int J Cancer 2002; 97: 403-9.

6. Minoo P, Zlobec I, Peterson M, et al. Characterization of rectal, proximal and distal colon cancers based on clinicopathological molecular and protein profiles. Int J Oncol 2010; 37: 707-18.

7. Brzozowa M, Mielańczyk L, Michalski M, et al. Role of Notch signaling pathway in gastric cancer pathogenesis. Contemp Oncol 2013; 17: 1-5.

8. Brzozowa-Zasada M, Piecuch A, Dittfeld A, et al. Notch signaling pathway as an oncogenic factor involved in cancer development. Contemp Oncol 2016; 20: 267-72.

9. Brzozowa-Zasada M, Piecuch A, Segiet O, et al. The complex interplay between Notch signaling and Snail1 transcription factor in the regulation of eithelial-mesenchymal transition. Eur Surg 2015; 47: 218-25.

10. Koch U, Radtke F. Notch and cancer: a double-edged sword. Cell Mol Life 2007; 64: 2746-62.

11. Borgrefe T, Oswald F. The Notch signaling pathway: transcriptional regulation at Notch target genes. Cell Mol Life Sci 2009; 66: 1631-46.

12. Kopan R, Ilagan MX. The canonical Notch signaling pathway: unfolding the activation mechanism. Cell 2009; 137: 216-33.

13. Brzozowa-Zasada M, Piecuch A, Michalski M, et al. Notch and its oncogenic activity in human malignancies. Eur Surg 2017; 49: 199-209.

14. Zhang Y, Li B, Ji ZZ, Zheng PS. Notch1 regulates the growth of human colon cancers. Cancer 2010; 116: 52017-18.

15. Chu D, Li Y, Wang W, et al. High level of Notch1 protein is associated with poor overall survival in colorectal cancer. Ann Surg Oncol 2010; 17: 1337-42. 
16. Fender AW, Nutter JM, Fitzgerald TL, et al. Notch-1 promotes stemness and epithelial to mesenchymal transition in colorectal cancer. J Cell Biochem 2015; 116: 2517-27.

17. Gopalakrishnan N, Sivasithamparam ND, Devaraj H. Synergistic association of Notch and NFKB signaling and role of Notch signaling in modulating epithelial to mesenchymal transition in colorectal adenocarcinoma. Biochimie 2014; 107 ptB: 310-8.

18. Kim HA, Koo BK, Cho JH, et al. Notch1 counteracts WNT/beta-catenin signaling through chromatin modification in colorectal cancer. J Clin Invest 2012; 122: 3248-59.

19. Kang H, An HJ, Song JY, et al. Notch3 and Jagged2 contribute to gastric cancer development and to glandular differentiation associated with MUC2 and MUC5 expression. Histopathology 2012; 61: 576-86.

20. Sun Y, Gao X, Liu J, et al. Differential Notch1 and Notch2 expression and frequent activation of Notch signaling in gastric cancers. Arch Pathol Lab Med 2011; 135: 451-8.

21. Wang Z, Li Y, Sarkar FH. Notch signaling proteins: legitimate targets for cancer therapy. Curr Protein Pept Sci 2010; 11 398-408.

22. Piazzi G, Fini L, Selgrad M, et al. Epigenetic regulation of DeltaLike 1 controls notch1 activation in gastric cancer. Oncotarget 2011; 2: 1291-301.

23. Mao XY, Wang XG, Lu XJ, et al. COX-2 expression in gastric cancer and its relationship with angiogenesis using tissue microarray. World J Gastroenterol 2007; 13: 2466-71.

24. Yeh TS, Wu CW, Hsu KW, et al. The activated Notch1 signal pathway is associated with gastric cancer progression through cyclooxygenase-2. Cancer Res 2009; 69: 5039-48.

25. Rizzo P, Miao H, D’Souza G, et al. Cross-talk between Notch and estrogen receptor in breast cancer suggests novel therapeutic approaches. Cancer Res 2008; 68: 5226-35.

26. Rose SL, Kunnimalaiyan M, Drenzek J, Seiler N. Notch1 signaling is active in ovarian cancer. Gynecol Oncol 2010; 117 130-3.

27. Fojo T. Multiple paths to a drug resistance phenotype:mutations, translocations, deletions and amplification of coding genes or promoter regions, epigenetic changes and microRNAs. Drug Resist Updat 2007; 10: 59-67.

28. Pogribny IP, Filkowski JN, Tryndyak VP, et al. Alterations of microRNAs and their targets are associated with acquired resistance of MCF-7 breast cancer cells to cisplatin. Int J Cancer 2010; 127: 1785-94.

29. Hwang JH, Voortman J, Giovanetti E, et al. Identification of microRNA-21 as a biomarker for chemoresistance and clinical outcome following adjuvant therapy in resectable pancreatic cancer. PLoS One 2010; 5: e10630.

30. Liu MX, Siu MKY, Liu SS, et al. Epigenetic silencing of microRNA-199b-5p is associated with acquyired chemoresistance via activation of JAG1-Notch1 signaling in ovarian cancer. Oncotarget 2014; 5: 944-58.

31. Donnem T, Andersen S, Al-Shibili K, et al. Prognostic impact of Notch ligands and receptors in nonsmall cell lung cancer: coexpression of Notch-1 and vascular endothelial growth factor-A predicts poor survival. Cancer 2010; 116: 5674-85.

32. Allen TD, Rodriguez EM, Jones KD, Bishop JM. Activated Notch1 induces lung adenomas in mice and cooperates with Myc in the generation of lung adenocarcinoma. Cancer Res 2011; 71: 6010-8.

33. Yuan X, Wu H, Xu H, et al. Meta-analysis reveals the correlation of Notch signaling with non-small cell lung cancer progression and prognosis. Sci Rep 2015; 5: 10338.

34. Westhoff B, Colaluca IN, D'Ario G, et al. Alterations of the Notch pathway in lung cancer. Proc Natl Acad Sci USA 2009; 106: 22293-8.

Received: 7.04.2019

Accepted: 22.11.2019 